\title{
Role of Coulomb correlations for femtosecond pump-probe signals obtained from a single quantum dot
}

\author{
J. Huneke, ${ }^{1,2}$ I. D’Amico, ${ }^{2}$ P. Machnikowski, ${ }^{3}$ T. Thomay, ${ }^{4}$ R. Bratschitsch, ${ }^{4}$ A. Leitenstorfer, ${ }^{4}$ and T. Kuhn ${ }^{1}$ \\ ${ }^{1}$ Institut für Festkörpertheorie, Universität Münster, Wilhelm-Klemm-Straße 10, DE-48149 Münster, Germany \\ ${ }^{2}$ Department of Physics, University of York, Heslington York YO10 5DD, United Kingdom \\ ${ }^{3}$ Institute of Physics, Wroctaw University of Technology, PL-50-370 Wrocław, Poland \\ ${ }^{4}$ Department of Physics and Center for Applied Photonics, University of Konstanz, DE-78457 Konstanz, Germany
}

(Received 25 January 2011; revised manuscript received 29 August 2011; published 22 September 2011)

\begin{abstract}
We present a theoretical analysis of femtosecond pump-probe experiments performed on a single negatively charged quantum dot. The influence of Coulomb-correlation effects as well as carrier relaxation on transient transmission change signals is investigated. Our model describes ultrafast disappearance of the fundamental trion absorption due to instantaneous Coulomb renormalizations and a delayed onset of gain at the same frequency, as found in the measurements. Going beyond previous experimental information, we predict that after spinconserving carrier relaxation, new optical transitions exhibiting either gain or absorption should emerge that build up on a picosecond timescale. The time dependence of these new transitions provides insight into details of the carrier relaxation processes.
\end{abstract}

DOI: $10.1103 /$ PhysRevB.84.115320

PACS number(s): 73.21.La, 78.47.J-, 78.67.Hc

\section{INTRODUCTION}

Optically driven semiconductor quantum dots (QDs) have been proposed as key components for few-particle devices, especially within quantum information processing schemes ${ }^{1-5}$ and as single photon sources. 6,7 Their ability of coupling solid-state degrees of freedom to light fields enables the possibility of ultrafast all-optical driving of the few-particle dynamics as well as the coherent transfer of (quantum) information. In this context, ultrafast coherent pump-probe spectroscopy is an excellent technique to study the fewfermion dynamics under the influence of Coulomb interactions and correlations. Pump-probe signals on QD ensembles have been widely studied, which provided insight into the dynamics of spin and orbital degrees of freedom. Depending on the excitation conditions and detection setup (hence on the type of the measured signal) the pump-probe technique provides a powerful tool to investigate both the charge kinetics (by measuring differential transmission or reflectivity) ${ }^{8-11}$ as well as the spin dynamics (by studying the Kerr or Faraday response $\left.\mathrm{e}^{12-16}\right)$. The inhomogeneous broadening, which is present in QD ensembles due to unavoidable size fluctuations, calls for an analysis of single QDs in order to obtain more detailed information on their physical properties and functionalities. Corresponding absorption measurements for single QDs and QD molecules as well as differential transmission and reflectivity measurements with picosecond light pulses have also been implemented, ${ }^{4,17-23}$ but the understanding of the interacting few-particle dynamics remains challenging.

Recently, two-color femtosecond pump-probe measurements on an individual, strongly confined and singly charged QD have been performed that allowed for an analysis of the dynamical behavior on a femtosecond timescale. ${ }^{24}$ Figure 1 shows a schematic illustration of the basic steps involved in that experiment. Initially the QD contains an electron in the lowest shell of the conduction band. A strong pump pulse tuned into resonance with a higher shell transition excites the QD. As a consequence, an electron-hole pair is generated that, together with the initially trapped electron, forms a three-particle state consisting of two electrons and one hole. Subsequently, both the excited electron and the hole relax to the ground state. Finally, they recombine leaving the QD again in its initial state. This final step, however, occurs on a much longer time scale than the other processes and will not be studied here. The dynamics in the QD were studied by measuring the transmission of a weak second pulse (the probe pulse), which was adjusted to the lowest energy-absorption line of the singly charged QD. ${ }^{24}$ The change in the transmission of this pulse caused by the pump pulse excitation was detected as a function of the delay time between pump and probe pulse. Within this experimental setup, the measurements revealed a strongly influenced probe pulse transmission signal undergoing pronounced changes when varying the delay time: for a probe pulse arriving before the pump pulse, i.e., for negative delay times, spectral oscillations on the energetically lowest transition line were observed. For short positive delay times between pump and probe pulse, a complete disappearance of the fundamental absorption line has been found. For longer positive delay times, the signal continuously reappeared; now, however, exhibiting optical gain. Already in Ref. 24, it has been suggested that these signals provide clear indications of the importance of Coulomb correlations of the few-particle states involved in the dynamics. However, the Coulomb-induced frequency shifts have not been monitored and the main mechanism perturbing the probe pulse signal and leading to its instantaneous disappearance remained unclear.

Motivated by the experiment described above, we present in this paper a theoretical analysis of such a pump-probe experiment in which the charge carrier kinetics is studied by measuring the differential transmission of the probe pulse in a pump-probe setup. In our study, we focus on the influence of Coulomb correlation effects and carrier relaxation on the fundamental transition line. For this purpose, we calculate the relevant Coulomb-correlated few-particle states and the 


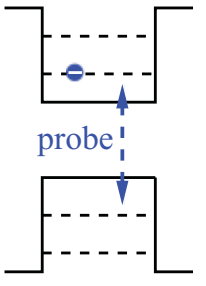

initial state

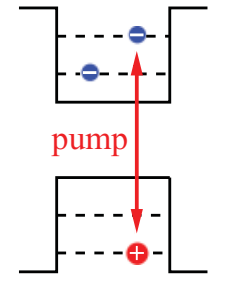

after pump pulse
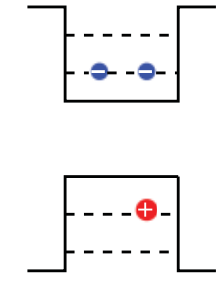

after relaxation
FIG. 1. (Color online) Schematic illustration of the analyzed pump-probe experiment. Initially the QD is charged with a single electron in the lowest conduction band state ( $s$ shell). The pump-pulse in resonance to a higher shell ( $p$ shell) creates an additional electronhole pair resulting in a trion in an excited state. Subsequently, the generated electron and hole relax to the $s$ shell. The pump-induced dynamics is monitored by the absorption of a probe pulse resonant on the $s$-shell transition.

corresponding oscillator strengths using a numerical diagonalization approach. The Coulomb-correlated few-particle states thus obtained are then used as the basis for solving the equations of motion that describe the dynamics induced by the pump and probe pulses, including the relaxation kinetics due to the interaction with the environment.

In this way, our theory not only accounts for the Coulombrelated spectral features that underlie the effects observed in the experiment but also provides a full description of the interplay between Coulomb correlations and relaxation kinetics, in particular of the transitions between various spin configurations of trions and charged biexcitons. In agreement with the previously measured signals, ${ }^{24}$ we find that the probed transition line is noticeably affected as soon as the pump pulse generates an electron-hole pair in a higher shell. By comparing our results with the case of noninteracting carriers we extract the role of Coulomb correlations and show that their absence leads to features that are in contradiction to the measurements. Furthermore, we predict additional spectral features, which have not yet been measured in this system and that could be used to confirm the model assumptions in the calculations.

The paper is organized as follows. The underlying theoretical model is presented in Sec. II. After this, in Sec. III, we discuss qualitatively the various steps of the pump-probe dynamics, which will allow us to identify the relevant fewfermion states involved in the experiment. Based on the spectral characterization of the QD states involved, presented in Sec. IV, we will then discuss spectrally resolved pumpprobe signals as a function of the time delay between pump and probe pulse (Sec. V). The paper then finishes with some concluding remarks.

\section{THEORY}

The Hamiltonian we use to model the experiment consists of two parts $H=H_{0}+H^{\prime}$. The first term, $H_{0}$, describes the subsystem of Coulomb-correlated electrons and holes in a strongly confined QD. It defines the spectral characteristics of the QD and will allow us to identify the energy states involved in the pump-probe experiment. The second term, $H^{\prime}$, induces the dynamics in the system. It is given by the coupling of the carrier system to a coherent light field (describing both pump and probe pulses) as well as to environmental degrees of freedom. In the following, we will discuss both contributions $H_{0}$ and $H^{\prime}$ separately.

\section{A. Coulomb-correlated carrier system}

When the QD is occupied by more than one electron or hole, Coulomb interactions couple different carriers. The resulting few-particle states are then eigenfunctions of the Hamiltonian $H_{0}=H_{c}+H_{c c}$. Therein, $H_{c}$ is the single particle Hamiltonian

$$
H_{c}=H_{c}^{e}+H_{c}^{h}=\sum_{i} \varepsilon_{i}^{e} c_{i}^{\dagger} c_{i}+\sum_{i} \varepsilon_{i}^{h} d_{i}^{\dagger} d_{i},
$$

where $c_{i}^{\dagger}$ and $d_{i}^{\dagger}\left(c_{i}\right.$ and $\left.d_{i}\right)$ are creation (annihilation) operators for electrons and holes with single particle energies $\varepsilon_{i}^{e}$ and $\varepsilon_{i}^{h}$, respectively. The index $i$ labels both orbital and spin degrees of freedom. We model the noninteracting carrier system in the effective-mass and envelope-function approximations and assume a quantum confinement given by an anisotropic harmonic oscillator potential. As is typical for self-assembled QDs, we assume that the confinement in the growth direction is much stronger than in the lateral directions so that only the lowest energy state in the growth $(z)$ direction is considered. The corresponding single-particle energies for electrons and holes $(\lambda=e, h)$

$$
\varepsilon_{n_{x}, n_{y}}^{\lambda}=\frac{1}{2} E_{G}+\hbar \omega_{x}^{\lambda}\left(n_{x}+\frac{1}{2}\right)+\hbar \omega_{y}^{\lambda}\left(n_{y}+\frac{1}{2}\right)+\hbar \omega_{z}^{\lambda} \frac{1}{2},
$$

with

$$
\hbar \omega_{j}^{\lambda}=\frac{\hbar^{2}}{m^{\lambda}\left(l_{j}^{\lambda}\right)^{2}},
$$

and $E_{G}$ denoting the energy gap of the bulk material are then determined by the confinement lengths $l_{j}^{\lambda}$ of the QD in the directions $j=x, y, z$ and the effective mass $m^{\lambda}$, which in the case of the holes, is assumed to be of heavy-hole type. The Hamiltonian describing the carrier-carrier interactions is given by

$$
\begin{aligned}
H_{c c}= & \frac{1}{2} \sum_{i j k l} V_{i j k l}^{e e} c_{i}^{\dagger} c_{j}^{\dagger} c_{k} c_{l}+\frac{1}{2} \sum_{i j k l} V_{i j k l}^{h h} d_{i}^{\dagger} d_{j}^{\dagger} d_{k} d_{l} \\
& -\sum_{i j k l} V_{i j k l}^{e h} c_{i}^{\dagger} d_{j}^{\dagger} d_{k} c_{l} .
\end{aligned}
$$

The Coulomb-interaction matrix elements read

$$
\begin{aligned}
V_{i j k l}^{\lambda \lambda^{\prime}}= & \int d^{3} r \int d^{3} r^{\prime} \Psi_{i}^{* \lambda}\left(\mathbf{r}^{\prime}\right) \Psi_{j}^{* \lambda \prime}(\mathbf{r}) V\left(\left|\mathbf{r}-\mathbf{r}^{\prime}\right|\right) \\
& \times \Psi_{k}^{\lambda^{\prime}}(\mathbf{r}) \Psi_{l}^{\lambda}\left(\mathbf{r}^{\prime}\right),
\end{aligned}
$$

with the Coulomb potential $V(|\mathbf{r}|)=e^{2} / 4 \pi \epsilon_{0} \epsilon_{r} r, \epsilon_{0}$ and $\epsilon_{r}$ being the vacuum and relative permittivity, respectively. The single-particle wave functions for electrons and holes are products of the eigenstates of one-dimensional harmonic oscillator potentials

$$
\Psi_{n_{x}, n_{y}}^{\lambda}(\mathbf{r})=\frac{1}{\sqrt{l_{x}^{\lambda} l_{y}^{\lambda} l_{z}^{\lambda}}} \Psi_{n_{x}}\left(\frac{x}{l_{x}^{\lambda}}\right) \Psi_{n_{y}}\left(\frac{y}{l_{y}^{\lambda}}\right) \Psi_{0}\left(\frac{z}{l_{z}^{\lambda}}\right)
$$


with

$$
\Psi_{n}(x)=\left(\frac{1}{\sqrt{\pi} 2^{n} n !}\right)^{\frac{1}{2}} H_{n}(x) e^{-\frac{1}{2} x^{2}}
$$

and $H_{n}(x)$ denoting the $n$th Hermite polynomial.

The Hamiltonian $H_{0}$ commutes with the total-number operator for electrons $N^{e}=\sum_{i} c_{i}^{\dagger} c_{i}$ and holes $N^{h}=\sum_{i} d_{i}^{\dagger} d_{i}$. It is therefore possible to calculate the Coulomb-correlated few-particle states for a given fixed number of fermions independently. Furthermore, we exploit the fact that the Coulomb interaction does not change the spin, so that apart from the total-number operators also the total spin of electrons and holes $S^{\lambda}$ and their corresponding spin projection $S_{z}^{\lambda}$ remain good quantum numbers. ${ }^{25-30}$

As will be discussed in Sec. III, the states relevant for the experimentally studied dynamics are the single-electron states $\left|e_{i}^{-}\right\rangle$, the trion states $\left|X_{\alpha}^{-}\right\rangle$, and the charged biexciton states $\left|X X_{\alpha}^{-}\right\rangle$, where $\alpha$ is an index that labels the Coulomb-correlated eigenstates of $H_{0}$. The single-electron states are eigenstates of $H_{0}$ with corresponding energies $\varepsilon_{i}^{e}$. The few-particle states $\left|X_{\alpha}^{-}\right\rangle$and $\left|X X_{\alpha}^{-}\right\rangle$can be expanded in a basis of uncorrelated electron and hole states as

$$
\begin{gathered}
\left|X_{\alpha}^{-}\right\rangle=\sum_{i j k} a_{i j k}^{(\alpha)} c_{i}^{\dagger} c_{j}^{\dagger} d_{k}^{\dagger}|0\rangle, \\
\left|X X_{\alpha}^{-}\right\rangle=\sum_{i j k l m} a_{i j k l m}^{(\alpha)} c_{i}^{\dagger} c_{j}^{\dagger} c_{k}^{\dagger} d_{l}^{\dagger} d_{m}^{\dagger}|0\rangle
\end{gathered}
$$

In order to obtain their expansion coefficients $a_{i j k}^{(\alpha)}$ and $a_{i j k l m}^{(\alpha)}$ and their eigenenergies $E_{X_{\alpha}^{-}}$and $E_{X X_{\alpha}^{-}}$, we perform a direct diagonalization of the Hamiltonian in a basis including up to the lowest six energy shells for electrons and holes of each in-plane direction, i.e., up to $n_{x}=n_{y}=5$. We truncate the basis at a fixed maximum energy for electrons and holes, i.e., $\varepsilon_{i}^{e}+\varepsilon_{j}^{e}<E_{C}$ and $\varepsilon_{k}^{h}<E_{V}$ for trions and $\varepsilon_{i}^{e}+\varepsilon_{j}^{e}+\varepsilon_{k}^{e}<E_{C}^{\prime}$ and $\varepsilon_{l}^{h}+\varepsilon_{m}^{h}<E_{V}^{\prime}$ for charged biexcitons. The cutoff energies $E_{C}, E_{V}, E_{C}^{\prime}$, and $E_{V}^{\prime}$ are chosen such that the energies of the lowest 25 trion states and 10 charged biexciton states have converged to less than $1 \mathrm{meV}$.

\section{B. Theoretical modeling of the pump-probe experiment}

The excitation of the QD with laser pulses induces a dynamical evolution that couples Coulomb-correlated states with different numbers of electrons and holes. We describe the interaction between the carriers and a classical electric field $\mathbf{E}$ in the usual dipole and rotating-wave approximations as

$$
H_{\mathrm{cl}}=-\left[\mathbf{E}^{(-)} \cdot \mathbf{P}+\mathbf{E}^{(+)} \cdot \mathbf{P}^{\dagger}\right] .
$$

Here, $\mathbf{P}$ is the interband dipole operator that recombines an electron-hole pair

$$
\mathbf{P}=\sum_{i j} \mathbf{M}_{i j} c_{i} d_{j}
$$

with the dipole matrix elements given by

$$
\mathbf{M}_{i j}=\mathbf{M}_{\mathrm{bulk}} \int \Psi_{i}^{e}(\mathbf{r}) \Psi_{j}^{h}(\mathbf{r}) d^{3} r,
$$

$\mathbf{M}_{\text {bulk }}$ being the bulk dipole matrix element between the valence band and the conduction band, and $\mathbf{E}^{(+)}\left[\mathbf{E}^{(-)}\right]$ denote the positive (negative) frequency part of the light field. Assuming the standard dipole selection rules only electrons and heavy-holes with spin projections having opposite signs are created or annihilated and the Hamiltonian couples only states differing by exactly one electron-hole pair.

The temporal evolution of the carrier system under the influence of this coupling to light is governed, in the densitymatrix formalism, by the Liouville-von Neumann equation

$$
i \hbar \dot{\rho}=\left[H_{0}+H_{c l}, \rho\right] .
$$

However, the system is not completely isolated and the additional coupling of the carrier subsystem with environmental degrees of freedom (mainly phonons) will lead to relaxation and decoherence processes. Here, we will not treat these interactions on a microscopic level and therefore we will not specify a Hamiltonian for this coupling. Instead, we will account for these various dissipative mechanisms via a collision (super)operator $\mathcal{L}$ taken to be of Lindblad-type that gives rise to the nonunitary evolution of the density matrix $\rho$ :

$$
\dot{\rho}=-\frac{i}{\hbar}\left[H_{0}+H_{c l}, \rho\right]+\mathcal{L}[\rho] .
$$

In the Born-Markov approximation, $\mathcal{L}$ is completely defined by the relaxation rates $\Gamma_{n n^{\prime}}$ for transitions from the state $\left|n^{\prime}\right\rangle$ to $|n\rangle$ and the associated dephasing rates $\Lambda_{n n^{\prime}}$ with $^{31,32}$

$$
\Lambda_{n n^{\prime}}=\frac{1}{2} \sum_{k}\left(\Gamma_{k n}+\Gamma_{k n^{\prime}}\right) .
$$

Then the resulting temporal evolution of the density matrix elements has the following form:

$$
\begin{aligned}
\dot{\rho}_{n n^{\prime}}= & -\frac{i}{\hbar}\left\langle n\left|\left[H_{0}+H_{c l}, \rho\right]\right| n^{\prime}\right\rangle+\sum_{k \neq n}\left(\Gamma_{n k} \rho_{k k}-\Gamma_{k n} \rho_{n n}\right) \delta_{n n^{\prime}} \\
& -\Lambda_{n n^{\prime}} \rho_{n n^{\prime}}\left(1-\delta_{n n^{\prime}}\right) .
\end{aligned}
$$

We solve these equations of motion to model the pump and probe pulse-induced dynamics using the calculated Coulomb correlated states $\left\{|n\rangle=\left|e_{i}^{-}\right\rangle,\left|X_{\alpha}^{-}\right\rangle,\left|X X_{\alpha}^{-}\right\rangle\right\}$as basis states. The pump-probe signal is given by the optical polarization induced by the probe pulse. We obtain this signal by calculating the total optical polarization as a function of the relative phase between pump and probe pulse and then extracting the probe contribution by Fourier transform following the method proposed in Ref. 33. A brief description of this method is given in Appendix. For the numerical results, we consider a single CdSe-based QD of ellipsoidal shape with characteristic lengths of the electron confinement potential $l_{x}^{e}=2.7 \mathrm{~nm}$, $l_{y}^{e}=2.5 \mathrm{~nm}$, and $l_{z}^{e}=l_{z}^{h}=1.0 \mathrm{~nm}$. The in-plane confinement lengths of holes and electrons are assumed to have a fixed ratio $\beta=l_{j}^{h} / l_{j}^{e}$, where the role of $\beta$ will be discussed in the next section. The conduction band effective mass is $m_{e}=0.13 m_{0}$, the valence band effective mass is $m_{h}=0.45 m_{0}, m_{0}$ being the free electron mass, and the relative permittivity is $\epsilon_{r}=9.57$, consistent with Ref. 34. The relaxation rates will be discussed in Sec. V. 


\section{FEW-PARTICLE STATES INVOLVED IN THE PUMP-PROBE DYNAMICS}

Before discussing the results of the numerical calculations let us have a closer look at the dynamics induced by the pump and the probe pulse for different delay times. This will allow us to identify the relevant few-particle states. For this purpose, in Fig. 2 we have schematically plotted the probe pulse-induced transitions and the corresponding carrier states at different delay times between pump and probe pulse. The lowest twoorbital states of the QD for both electrons and holes ( $s$ shell and $p$ shell) are taken into account. Both pump and probe pulses are linearly polarized where the probe pulse is centered to the $s$ shell while the pump pulse is centered to the $p$ shell. The left column depicts the dynamics induced by the pump pulse, the right columns show the final states after transitions that are induced by a probe pulse at various steps of the pumpinduced dynamics. For simplicity, in this qualitative discussion we neglect the correct antisymmetrization of the orbital and spin states, which, however, is of course correctly taken into account in the calculations.

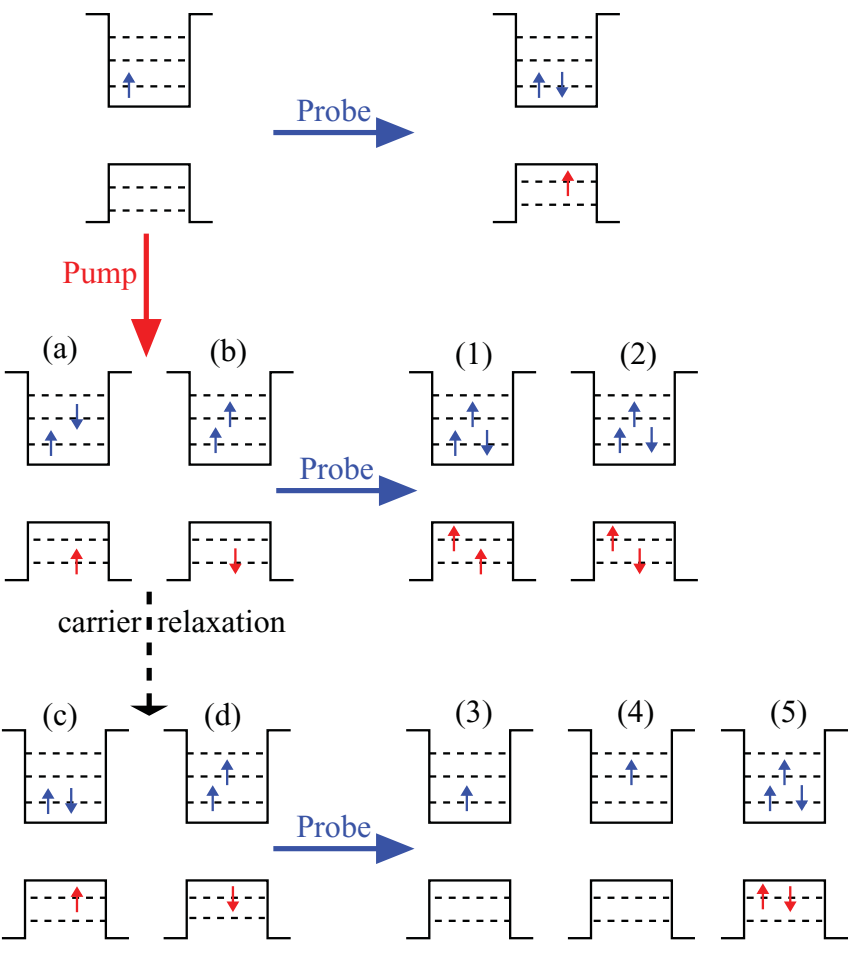

FIG. 2. (Color online) Schematic illustration of the analyzed pump-probe experiment. Initially, the QD is charged by a single electron in the lowest conduction band state ( $s$ shell). At time $t=0$, the pump-pulse excites an electron-hole pair in the $p$ shell, which subsequently relaxes toward the $s$ shell (left column). The various steps are monitored by a probe pulse on the $s$-shell transition. At negative delay times the probe pulse creates a trion in its ground state. Immediately after the pump pulse, it leads to optical transitions to an excited charged biexciton [states (1) and (2)]. After spin-conserving carrier relaxation, new optical transitions emerge resulting in stimulated emission to a single-electron state [states (3) and (4)] and absorption to a charged biexciton in the ground state [state (5)].
At negative delay times the probe pulse excites the QD in its ground state, i.e., charged by a single electron in the $s$ shell. The probe pulse is absorbed leading to the generation of a negatively charged exciton, i.e., a trion in its ground state. At time $t=0$, the pump pulse excites an electron-hole pair in the $p$ shell; for a linearly polarized pump pulse, the spin configurations indicated in Fig. 2 as (a) or (b) are both populated with equal probabilities. The subsequent absorption of the probe pulse leads to the creation of five-particle complexes consisting of three electrons and two holes, i.e., negatively charged biexcitons. These charged biexcitons are in an excited state because one hole is in the $s$ shell and the other in the $p$ shell.

The pump-induced excited trions start to relax toward their ground state. This relaxation, however, involves different time scales. Typically the relaxation of the holes is the fastest process. Slightly slower is the relaxation of the electron, as long as there is no spin flip involved. Both processes occur on a time scale of a few picoseconds and lead to the ground-state trion indicated by (c) in Fig. 2. Spin-flip processes of electrons typically occur on a much longer time scale, therefore on the picosecond time scale studied here excited trions with parallel spin configurations only experience a hole relaxation ending up in the state (d). In the case of state (c), the incoming probe photon leads to a stimulated emission by annihilating one electron-hole pair in the QD. Thus we expect to see gain instead of absorption in the probe transition. In the case of state (d), the linearly polarized probe pulse may either induce a stimulated emission process leading to the excited single-electron state (4) or an absorption process leading to the charged biexciton in its ground state (5).

From this qualitative description we can deduce the fewparticle states that are required to model both the pumpinduced dynamics and the probe-induced transitions. In addition to the single-electron states, we need a variety of negatively charged trion and negatively charged biexciton states. Without Coulomb interaction, their energies were simply given by the sum of the corresponding single-particle energies. As a consequence, the energy of the probe-induced transitions would always be the same, and we would simply expect a bleaching when the final state becomes occupied and eventually a change of the sign indicating gain due to stimulated emission. The Coulomb interaction, on the other hand, will lead to correlation effects that shift the transition energies and also modify the oscillator strengths resulting in a much more complex evolution of the pump-probe spectra. Before modeling the carrier dynamics in the QD, in the next section, we will analyze the spectral characteristics of the various pump or probe-induced transitions.

\section{SPECTRAL CHARACTERIZATION}

The specific characteristics of few-particle eigenstates in self-assembled QDs strongly depend on the geometry, size and material composition of the dot. ${ }^{35-39}$ Nevertheless it has been found that some general features in the optical spectra can be traced back to well-defined physical parameters. Both eight-band $\mathbf{k} \cdot \mathbf{p}$ calculations ${ }^{36,38}$ and advanced atomistic models ${ }^{35,37}$ have shown that in particular the relative magnitude of the Coulomb matrix elements representing 
particle-particle repulsion ( $V^{e e}$ and $V^{h h}$ ) and attraction $\left(V^{e h}\right)$ play a dominant role for the relative spectral positions of different few-particle complexes. Since the Coulomb matrix elements depend on the wave functions of electrons and holes, it is ultimately the difference in the extension of the electron and hole wave functions and their corresponding overlap that defines these spectral positions. By varying the ratio $\beta$ between the characteristic lengths of the hole and the electron wave functions, our model allows us to reproduce the following important situations assuming that the electron and hole wave functions are not strongly spatially separated; ${ }^{38}$ if the hole wave function is stronger (weaker) localized than the electron wave function, i.e., $\beta<1(\beta>1)$, then $V_{h h}>V_{e h}>V_{e e}\left(V_{h h}<V_{e h}<V_{e e}\right)$, which makes the hole-hole Coulomb-repulsion stronger (weaker) with respect to the electron-electron Coulomb repulsion.

Let us first discuss the impact of the parameter $\beta$ on the optical transitions from the ground state of the negatively charged QD to a charged-exciton (trion) state with two electrons and one hole. We evaluate the optical absorption using Fermi's golden rule and account for various line broadening mechanisms by replacing the $\delta$ function in Fermi's golden rule by a Lorentzian. In Figs. 3(a)-3(c), the calculated absorption spectrum up to $180 \mathrm{meV}$ (corresponding to the $\sim 30$ lowest trion states) is plotted for three different values of $\beta$. The energies are given relative to the fundamental trion line (FTL). All three absorption spectra are qualitatively similar showing three main absorption lines. Our numerical results indicate that the states corresponding to these three lines are dominated by a single electron-hole state in the noninteracting basis with only weak admixtures of other states. Since the confinement lengths in the in-plane directions $x$ and $y$ are similar, the single-particle states are still organized in shells referred to as $s, p, \ldots$, where $s$ shell means $n_{x}+n_{y}=0$ and $p$ shell corresponds to $n_{x}+n_{y}=1$. The line at $E=0 \mathrm{meV}$ shows the optically allowed FTL between the ground state, where a single electron is present, and the lowest trion state, where all particles are in the $s$ shell. In this $s$-shell trion state, the electrons have opposite spins and form a singlet state (the state is $\left|1 e_{\uparrow}^{s} 1 e_{\downarrow}^{s} 1 h_{\Downarrow}^{s}\right\rangle$ or $\left.\left|1 e_{\uparrow}^{s} 1 e_{\downarrow}^{s} 1 h_{\Uparrow}^{s}\right\rangle\right)$. The other two lines, which have a similar strength as the FTL, originate from $p$-shell transitions, which are split due to the anisotropic in-plane confinement potential. The electrons of the resulting excited trion have a triplet spin configuration and the three-particle state can be labeled by the projection of the total angular momentum on the $z$ direction $S_{z}$,

$$
\begin{gathered}
\left|S_{z}=\frac{3}{2}\right\rangle=\frac{1}{\sqrt{2}}\left(\left|1 e_{\downarrow}^{s} 1 e_{\uparrow}^{p}\right\rangle+\left|1 e_{\uparrow}^{s} 1 e_{\downarrow}^{p}\right\rangle\right)\left|1 h_{\Uparrow}^{p}\right\rangle, \\
\left|S_{z}=-\frac{3}{2}\right\rangle=\frac{1}{\sqrt{2}}\left(\left|1 e_{\downarrow}^{s} 1 e_{\uparrow}^{p}\right\rangle+\left|1 e_{\uparrow}^{s} 1 e_{\downarrow}^{p}\right\rangle\right)\left|1 h_{\Downarrow}^{p}\right\rangle, \\
\left|S_{z}=\frac{1}{2}\right\rangle=\left|1 e_{\downarrow}^{s} 1 e_{\downarrow}^{p}\right\rangle\left|1 h_{\Uparrow}^{p}\right\rangle, \\
\left|S_{z}=-\frac{1}{2}\right\rangle=\left|1 e_{\uparrow}^{s} 1 e_{\uparrow}^{p}\right\rangle\left|1 h_{\Downarrow}^{p}\right\rangle .
\end{gathered}
$$

Within each of the lines from the $p$-shell transition, these four states are degenerate. However, for a given initial spin orientation of the electron in the $s$ shell, only two of them can be reached due to the selection rules.
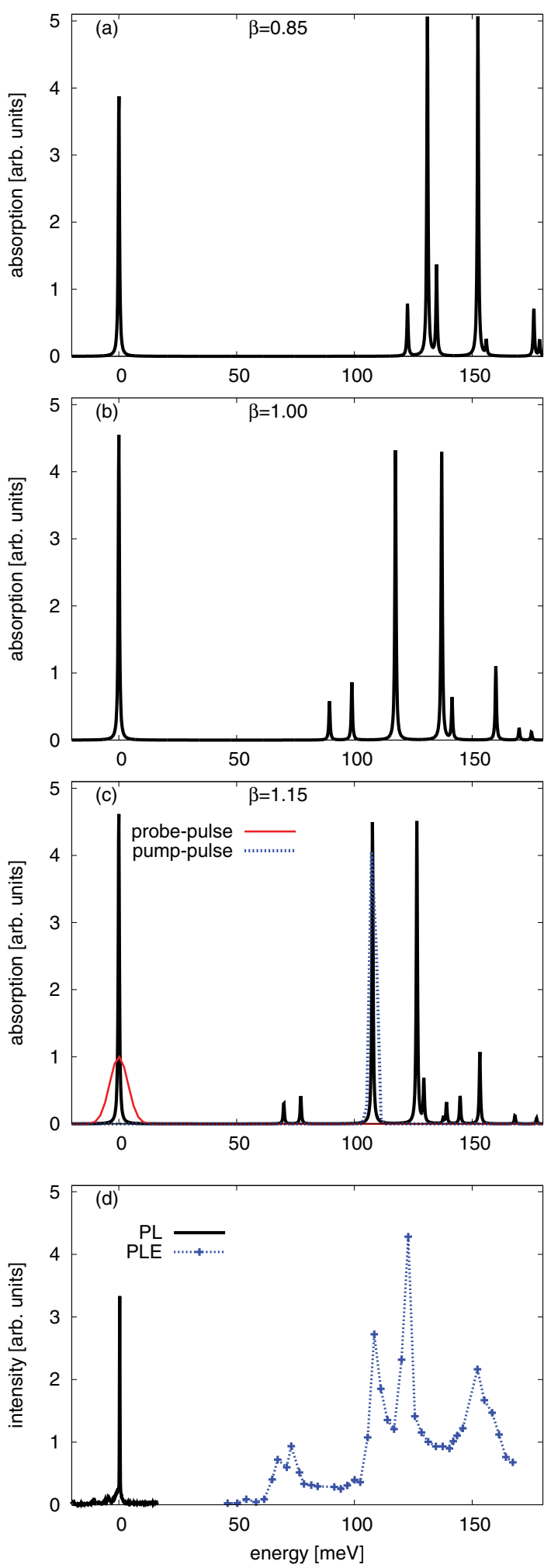

FIG. 3. (Color online) (a)-(c) Calculated trion absorption spectra of a singly charged quantum dot. Energies are given with respect to the lowest energy transition. The ratio $\beta$ between the confinement length of holes and electrons is taken as (a) $\beta=0.85$, (b) $\beta=1.00$, and (c) $\beta=1.15$. (d) Measured PL and PLE spectra of the singly charged QD used in the two color pump-probe experiment in Ref. 24. In (c), also the spectral envelopes of the pump pulse (dashed blue line) and probe pulse (thin red line) as used in the experiment in Ref. 24 are depicted. 
Around these main excited transition lines a number of weaker absorption lines appears that correspond to strongly mixed singlet states. Between the FTL and the first triplet peak, two rather weak peaks appear, which shift for decreasing $\beta$ in between the two triplet absorption peaks. These lines can be associated with transitions to nominally forbidden singlet states where both electrons are in the $s$ shell, while the hole is in the $d$ shell, ${ }^{40}$ i.e., $\left|1 e_{\uparrow}^{s} 1 e_{\downarrow}^{s} 1 h_{\Downarrow}^{d}\right\rangle$ or $\left|1 e_{\uparrow}^{s} 1 e_{\downarrow}^{s} 1 h_{\Uparrow}^{d}\right\rangle$. These transitions become optically active due to Coulombmixing effects between different singlet states and reveal the enhancement of Coulomb interactions in small QDs. As a consequence of the conservation of the total electron spin $S^{e}$, singlet and triplet states do not mix. This fact explains the weak admixture of other states to the triplet states resulting in pronounced absorption lines. It should be noted that typically the degeneracy of the triplet states is lifted in anisotropic QDs due to exchange interactions between electrons and holes and due to spin-orbit effects on electron-electron interactions. ${ }^{41-43}$ However, the resulting splittings are relatively small and beyond the spectral resolution determined by the subpicosecond laser pulses as well as the short lifetime of the excited trion state caused by the carrier relaxation times. Therefore they have been neglected in our model.

The findings are generic for all trion absorption spectra discussed here although the specific position and oscillator strength of the individual lines noticeably depend on the chosen value of $\beta$. In order to fix $\beta$ we compared our calculated spectra with the photoluminescence (PL) and the photoluminescence excitation (PLE) measurements performed in Ref. 24. The corresponding experimental spectra are presented in Fig. 3(d) and the comparison reveals the best qualitative and quantitative agreement for $\beta=1.15$ [see Fig. 3(c)]. This choice of $\beta$ corresponds furthermore to the physical assumption that the confinement potentials of electrons and holes are scaled by a fixed ratio, which is given by the ratio between conduction band offset and valence band offset for barrier ( $\mathrm{ZnSe}$ ) and QD material (CdSe). ${ }^{44,45}$ This correspondence between the results we obtain, and the physical assumption further confirms that the parabolic confinement model used for our calculation captures the relevant features of the QD structure.

The good agreement between calculated and measured spectra allows us to identify the electronic states, which were resonantly excited and probed in the pump-probe experiment. In agreement with the experimental conditions in our calculations, the probe pulse will be tuned to the FTL transition and the pump pulse to the lower one of the two $p$-shell triplet transitions. For reference, we have added in Fig. 3(c) the spectral envelopes corresponding to the $700 \mathrm{fs}$ pump pulse (dashed blue line) and the $180 \mathrm{fs}$ probe pulse (thin red line), as used in Ref. 24.

Having identified the energy shell that is resonantly excited in the pump-probe experiment, we may now discuss probe pulse induced transitions starting from that specific trion state. The corresponding transitions in the noninteracting particle picture are those shown in Fig. 2, which have already been briefly discussed. Immediately after the pump pulse, the QD is in one of the trion states schematically depicted by (a) and (b). The subsequent probe pulse centered on the $s$ shell then induces transitions to a charged biexciton with one hole in

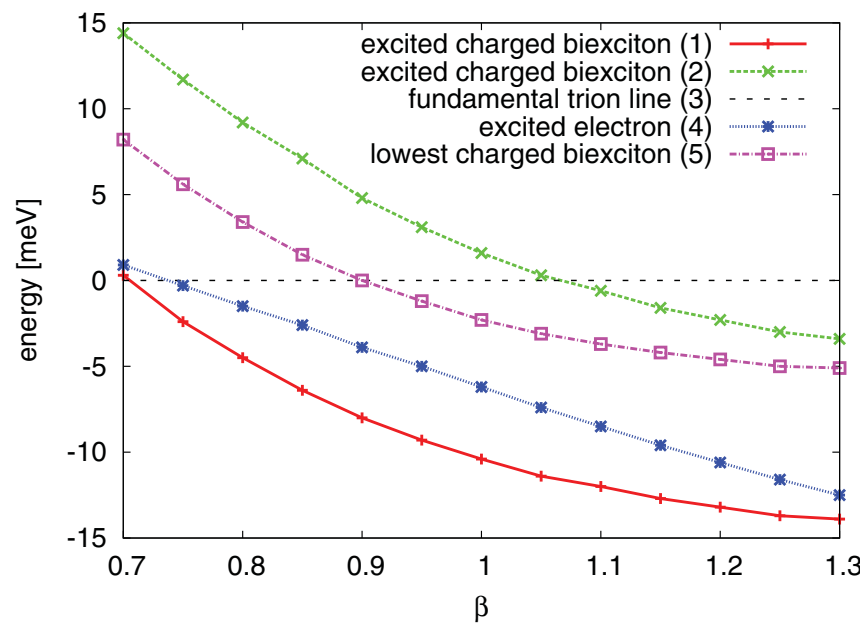

FIG. 4. (Color online) Calculated spectroscopic shifts of the optical transitions starting from a trion state to the states shown schematically in Fig. 2 as a function of $\beta$. The energies are given with respect to the energy of the FTL.

the $s$ shell and the other hole in the $p$ shell. After carrier relaxation, the trion states denoted as (c) and (d) are populated and a subsequent probe pulse will experience either gain to the single-electron states (3) and (4) or absorption to the charged biexciton (5). While, for noninteracting carriers, all five transitions leading to the states (1) to (5) had the same energy, now they experience different Coulomb correlations and are therefore split in energy.

The relative spectral positions of these transition lines with respect to the FTL are plotted in Fig. 4 as a function of the parameter $\beta$. If the hole wave function is localized, more strongly than the electron wave function, i.e., $\beta<1$, one deals with $V_{h h}>V_{e h}>V_{e e}$. This imbalance, strengthening the hole-hole Coulomb-repulsion with respect to the electronelectron Coulomb-repulsion, generally results in antibinding charged biexciton states. A weaker localization of the hole wave function with respect to the electron wave function on the other hand results in $V_{h h}<V_{e h}<V_{e e}$ and leads to binding charged biexcitons with respect to the FTL. It is worth noting that the crossing from binding to antibinding does not necessarily occur at $\beta=1$. Carrier correlations and exchange interactions affect the overall energy as well, where a parallel spin configuration reduces the Coulomb-repulsion due to the Pauli exclusion principle. As a consequence, we find for equally localized carrier wave functions $(\beta=1)$, a binding energy of $\sim 2 \mathrm{meV}$ for the lowest charged biexciton state. There is no experimental information on the spectral position of the charged biexciton in the single QD studied in the pump-probe experiment. However, PL measurements on single self-assembled CdSe QDs with similar trion PLE spectra have reported a systematic red-shift of the charged biexciton line of about $5-9 \mathrm{meV},{ }^{46}$ which is consistent with our calculation for $\beta=1.15$ giving a red-shifted charged biexciton of $\sim 4 \mathrm{meV}$.

\section{PUMP-PROBE DYNAMICS}

In this section, we now study the dynamics induced by the pump and probe pulses in combination with carrier relaxation. 
We start with a statistical mixture of the two singly charged states where both electron spin states are occupied with equal probabilities. The pump pulse has a duration of $700 \mathrm{fs}$ and a pulse area of $\pi$, i.e., it excites one electron-hole pair in the $p$ shell. The probe pulse, with a duration of $180 \mathrm{fs}$, has a pulse area of $\pi / 100$ such that the response remains well within the linear regime. The resonant excitation by the pump pulse into a higher shell creates an excited trion where the electron spins form a triplet state. Since the pump pulse excites the QD with a linear polarization, the resulting excited triplet state will consist of a combination of all four states defined in Eqs. (17)-(20). Therein, states with the projection of the total electron spin $S_{z}^{e}= \pm 1$, i.e., the states given in Eqs. (19) and (20), will be populated twice stronger due to a two times stronger oscillator strength. Immediately after the optical initialization, cooling processes cause carrier relaxation toward the lowest trion ground state [(c) in Fig. 2]. The times for spin-conserving energy relaxation processes are typically of the order of several picoseconds. ${ }^{47,48}$ Energy relaxation occurring exclusively in the valence band, i.e., hole relaxation, has no restrictions regarding spin conservation. Therefore, we assume a hole relaxation time of $\tau_{h}=3 \mathrm{ps}$ for the path from the optically excited triplet state to the lowest triplet state. In the case of the electron relaxation, one deals with intraband transitions from a triplet to a singlet state. For electrons with $S_{z}^{e}= \pm 1$, this requires spin-flip processes, which are strongly suppressed in a QD. ${ }^{10,49}$ Also transitions from the triplet state with $S_{z}^{e}=0$ [see Eqs. (17) and (18)] should in principle be forbidden as they require a change of the electron total angular momentum from 1 to 0 . However, in Ref. 40, a possible relaxation path through a singlet-triplet coupling mediated by anisotropic electron-electron interactions ${ }^{50-52}$ has been discussed and a relaxation time differing by one order of magnitude between the two triplet configurations has been estimated based on power-dependent PLE-measurements. This is in very good agreement with the experimental findings in Ref. 24. Hence, we take two phenomenological relaxation times in accordance with the experimental findings and choose for electrons with $S_{z}^{e}=0$ [states given by Eqs. (17) and (18)] $\tau_{e}=12 \mathrm{ps}$ and for electron relaxation processes involving spin-flip processes [states given by Eqs. (19) and (20)], $\tau_{\text {spin flip }}=150$ ps.

Figure 5 shows the temporal evolution of the QD occupation probabilities after the pump-pulse excitation applied at $t=0$ on the $p$-shell transition. The occupation of the optically initialized trion states (A) and (B) decreases after the excitation, while the occupations of states with intermediate energies increase. After the relaxation of both the electron and the hole, also the trion ground state becomes occupied. As spin-flip transitions from the trion states with $S_{z}^{e}= \pm 1$ occur on a much longer time scale, the trion ground-state occupation probability increases only up to slightly above $1 / 3$ resulting almost exclusively from the relaxation of the $S_{z}^{e}=0$ triplet trion.

The interplay between Coulomb correlations and intraband carrier relaxation can be best monitored by analyzing the probe pulse-induced absorption. In order to clearly determine the role of Coulomb correlations, let us first have a look at probe spectra as obtained from a theoretical simulation without taking into account Coulomb correlations. The resulting spectra are shown in Fig. 6(a) as a function of the delay time $\tau$ between the pump and probe pulse where the excitation scenario corresponds

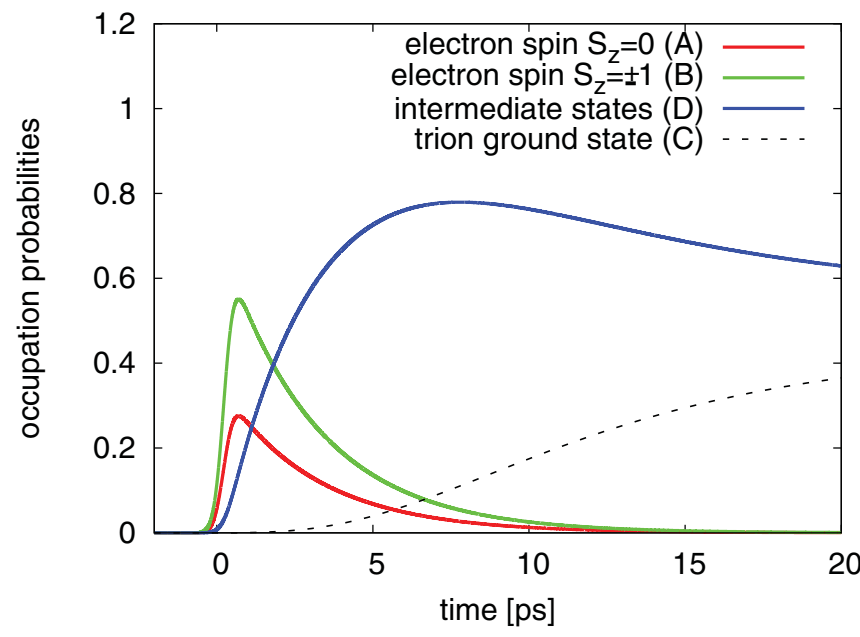

FIG. 5. (Color online) Simulated temporal evolution of the trion state occupation probabilities generated by a $\pi$-pulse excitation with a $700 \mathrm{fs}$ pulse on the $p$-shell transition at $t=0 \mathrm{ps}$. Labels refer to the states shown in Fig. 2.

to the discussion in Sec. III. The calculated spectra reveal a gradual change from an absorption line at sufficiently long negative delay times to a gain line at long positive delay times, which reflects the fact that without Coulomb correlations the energy of the probe-induced transitions is always the same. The probe pulse signal is first reduced by Pauli blocking and eventually it evolves into gain after carrier relaxation into the state (c) or (d). In the case of the state (c), the incoming probe photon leads to a stimulated emission by annihilating one electron-hole pair in the QD. In the case of the state (d), there may be either stimulated emission leading to the state (4) or absorption leading to the five-particle state (5). For a linearly polarized probe pulse, these latter two processes cancel exactly. Thus the gain is expected to be only half as large as the initial absorption. Except for some small deviations resulting from the fact that even after $20 \mathrm{ps}$ the relaxation for the triplet state with $S_{z}^{e}=0$ is not yet completely finished, this scenario is indeed seen in Fig. 6(a).

When comparing these spectra with the corresponding results taking into account Coulomb correlations [see Fig. 6(b)], the importance of these correlations is clearly evident. In contrast to a single line, which only changes its height and sign from absorption to gain, now we see a whole series of lines exhibiting either absorption or gain. Different lines, in general, exhibit quite different delay-time dependencies reflecting the details of the relaxation processes. In the following, we will discuss the different temporal regimes.

\section{A. Negative delay times}

When the probe pulse comes before the pump pulse, the signal shows a single absorption line on the FTL that is surrounded by pronounced spectral oscillations. The frequency of these oscillations corresponds to the delay time between pump and probe pulse. This coherent effect is typical for spectrally resolved excitations on narrow transition lines and can be explained as follows: the probe pulse first creates a coherent polarization at the lowest trion energy. The following strong pump pulse then creates an excited electron-hole pair 
(a)

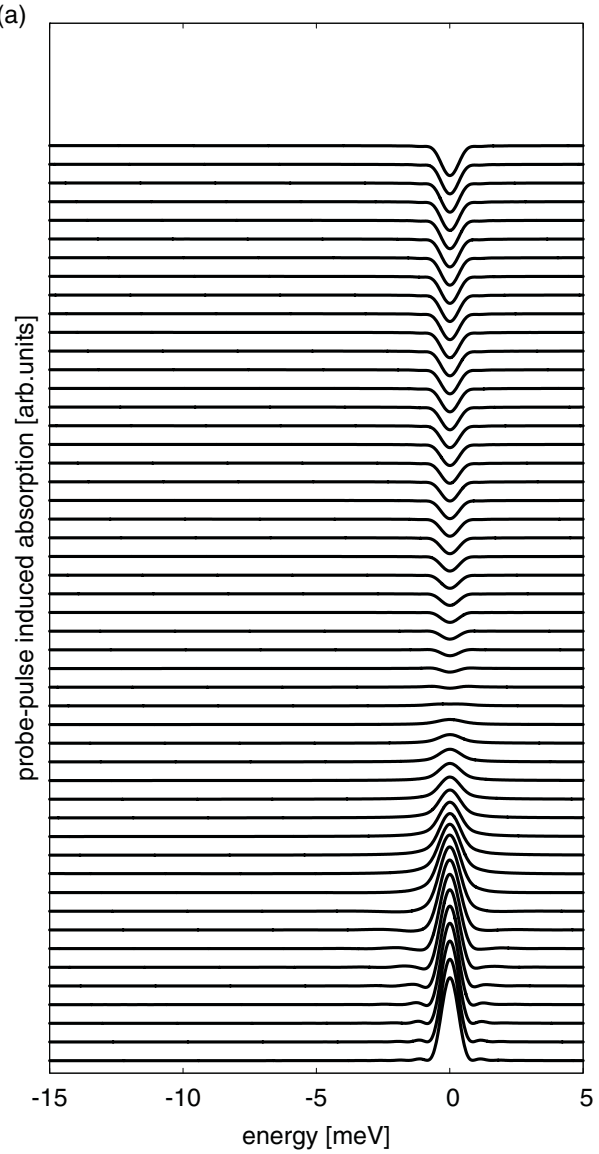

(b)

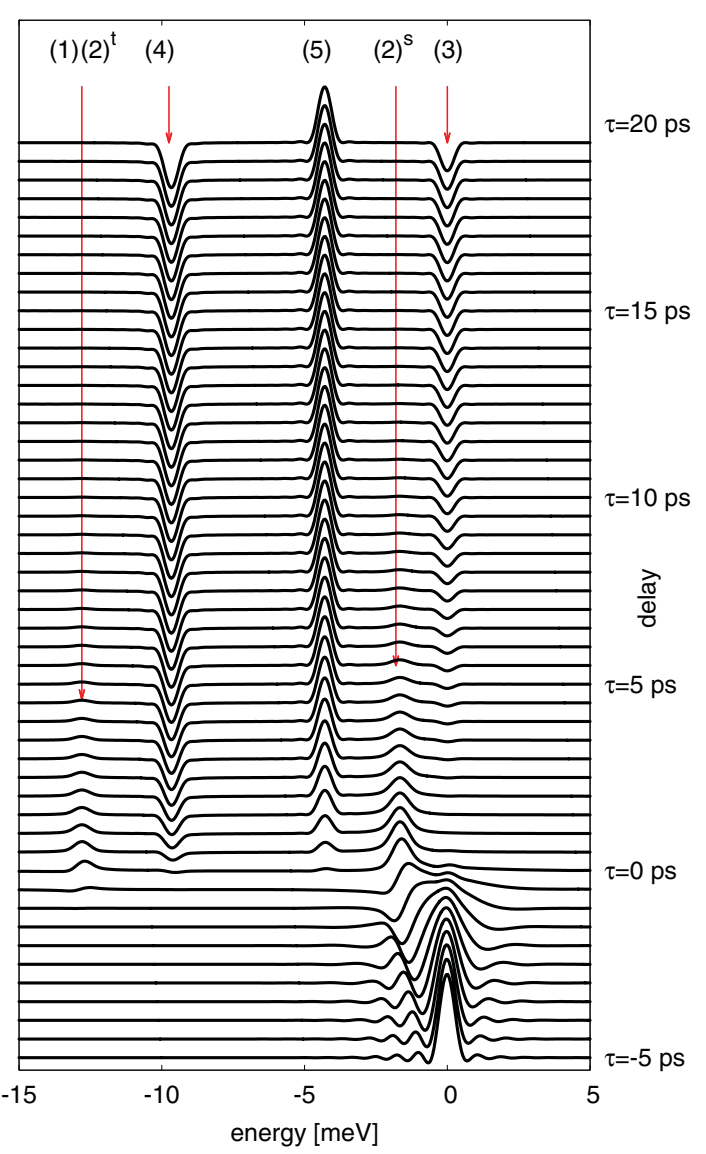

FIG. 6. (Color online) Probe-pulse absorption spectra calculated as a function of energy and delay time $\tau$ between pump and probe-pulse: (a) for the case of noninteracting carriers and (b) including Coulomb interactions. The pump pulse is in resonance to the lowest $p$-shell transition (see Fig. 2). The energy is measured with respect to the fundamental trion line. The labels (1)-(5) refer to the electron and charged biexciton states shown schematically in Fig. 2. Here, $(2)^{t}$ corresponds to the holes in a triplet state, while $(2)^{s}$ to the holes in a singlet state.

causing an instantaneous renormalization of the energy of the FTL and thereby perturbs the free induction decay of the probe pulse-induced polarization. In a spectrally resolved signal, such abrupt changes in the time domain are reflected as spectral oscillations. In the pump-probe spectra without Coulomb interactions [see Fig. 6(a)], such spectral oscillations are present as well, but they are extremely weak. There the perturbation of the free induction decay results only from the hole relaxation: when the hole relaxes, the excited trion state consists of one electron and one hole in the $s$ shell and one electron in the $p$ shell $\left(1 e^{s} 1 e^{p} 1 h^{s}\right)$ so that depending on the spin configurations of electrons and holes, either a bleaching of the transition, an absorption to a charged biexciton $\left(2 e^{s} 1 e^{p} 2 h^{s}\right)$, or a stimulated emission to a single electron $\left(1 e^{p}\right)$ may occur on the FTL [see states (4) and (5) in Fig. 2]. As a result, the probe pulse polarization decreases with an increasing occupation of trion states having a hole in the lowest shell $\left(1 e^{s} 1 e^{p} 1 h^{s}\right)$. In our model, this decay is exponential with a decay time of 3 ps given by the hole relaxation.

To demonstrate the different temporal behavior of the probe polarization in the presence or absence of Coulomb renormalizations, we have plotted in Fig. 7 the height of the absorption spectrum at the position of the FTL as a function of the delay time for both cases. Indeed, we find that without
Coulomb renormalization the absorption gradually decreases around $\tau=0$ and vanishes at about 5 ps. In contrast, in the presence of Coulomb renormalizations, the absorption at the FTL energy vanishes already at $\tau=0$ because the pump-excited trion, although not occupying any of the states involved in the FTL transitions, shifts this transition out of resonance. This gives rise to the rather abrupt change in the pump-probe signal at $\tau=0$ clearly seen in Fig. 6(b).

Spectral oscillations in pump-probe signals at negative delay times have been observed in many different systems. $^{22,23,53-55}$ They have often been modeled by including a phenomenological density-dependent dephasing rate. ${ }^{22,56,57}$ This is similar to our case without Coulomb interaction. However, we find that in our system the Coulomb-induced frequency shifts strongly dominate the perturbed free induction decay. The rather weak polarization decay for the case without Coulomb correlations results in much less pronounced spectral oscillations.

\section{B. Positive delay times}

Let us now come back to the full probe spectra and discuss their behavior at positive delay times. Here, these Coulomb renormalization effects can be directly seen by the 


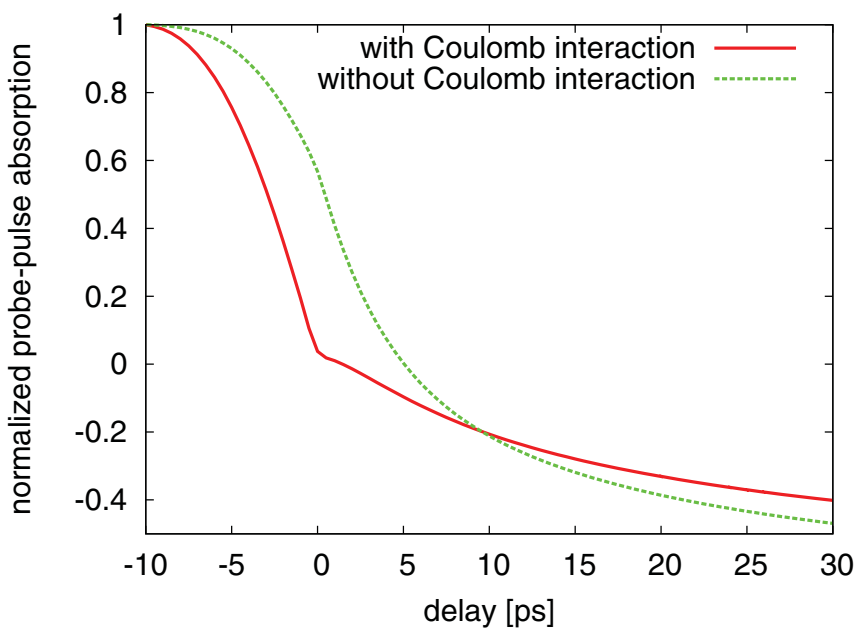

FIG. 7. (Color online) Amplitude of the fundamental trion absorption line (FTL) as a function of delay time $\tau$ between pump and probe pulse with Coulomb interaction (red solid line) and without Coulomb interaction (green dashed line).

appearance of new lines. We find that the probe pulse-induced absorption now splits into five new optically active resonances, which display the transitions discussed in Fig. 2. Accordingly, they are labeled by the corresponding final states (1)-(5) in that figure. Let us first discuss the absorption lines, i.e., the transitions from the trion to a charged biexciton. When no carrier relaxation has taken place, transitions to an excited charged biexciton are induced where one hole is in the lowest energy shell and one hole in a higher energy shell. The corresponding lines are labeled (1), (2) ${ }^{t}$, and $(2)^{s}$. In the state (2), the holes have different spin orientations, therefore they either form a singlet state $(2)^{s}$ with $S_{h}=0$ or a triplet state $(2)^{t}$ with $S_{h}=3$. The latter has the same energy as the triplet state (1). Singlet and triplet states are separated by $\sim 11 \mathrm{meV}$. Indeed, it turns out that this energy separation is close to the hole exchange matrix element $V_{s p s p}^{h h} \sim 12 \mathrm{meV}$ between holes in $s$ and $p$ states. Hence, the reported vanishing FTL may be traced back at least in part to strong exchange interactions between holes in different shells. The amplitudes of the two absorption lines are weak and decay fast due to fast hole relaxations that start immediately after the creation of the excited trion (see Fig. 5).

If the probe pulse reaches the QD after the hole relaxation, a transition to a charged biexciton is induced where both holes are in the lowest shell. Here, hole-hole exchange interactions can no longer split the charged biexciton spectra so that only one absorption line corresponding to the fundamental charged biexciton transition appears [labeled (5)]. Its build-up as a function of delay time is related to the hole relaxation time and its amplitude remains almost constant for larger delay times with a value of about $2 / 3$ of the fundamental trion absorption amplitude without pump pulse perturbation. Hence, its amplitude gives access to the trion occupation. As the occupation of trions with electrons in the $S_{z}^{e}= \pm 1$ spin configuration decays very slowly, the corresponding transitions remain prominent at large delay times.

The two transition lines with a negative sign describe gain and therefore monitor the dynamics from the trion state back to the single negatively charged QD. The transition line labeled (4) is strongly red-shifted with respect to the fundamental trion line and is associated with a transition where the final state is a single electron in a higher energy shell. The strong shift can be explained by the electron-electron exchange interaction that considerably lowers the Coulomb repulsion. This line is to some extent the counterpart of the absorption line corresponding to the charged biexciton transition (5). After a rapid build-up following the hole relaxation, its amplitude remains almost constant and decays very slowly in accordance with the slow spin-flip relaxation time. Again, its amplitude is about $2 / 3$ of the fundamental trion absorption.

For large positive delays, the hole as well as the electron that had been created in the $S_{z}^{e}=0$ triplet state have relaxed to the lowest trion state. In this case, the probe pulse gives rise to a stimulated emission to recover a QD with a single electron in the lowest energy shell. This is exactly the inverse process of the fundamental trion absorption, so that the corresponding spectral resonance resides at the same energy $E=0$. Thus a line at the position of the FTL reappears, now however exhibiting gain. When looking at the amplitude of the spectra at $E=0$ as a function of the delay time (see Fig. 7), we find that at longer times, the curves resulting from the calculations with and without Coulomb correlations approach each other. However, a full agreement of these lines will only be reached
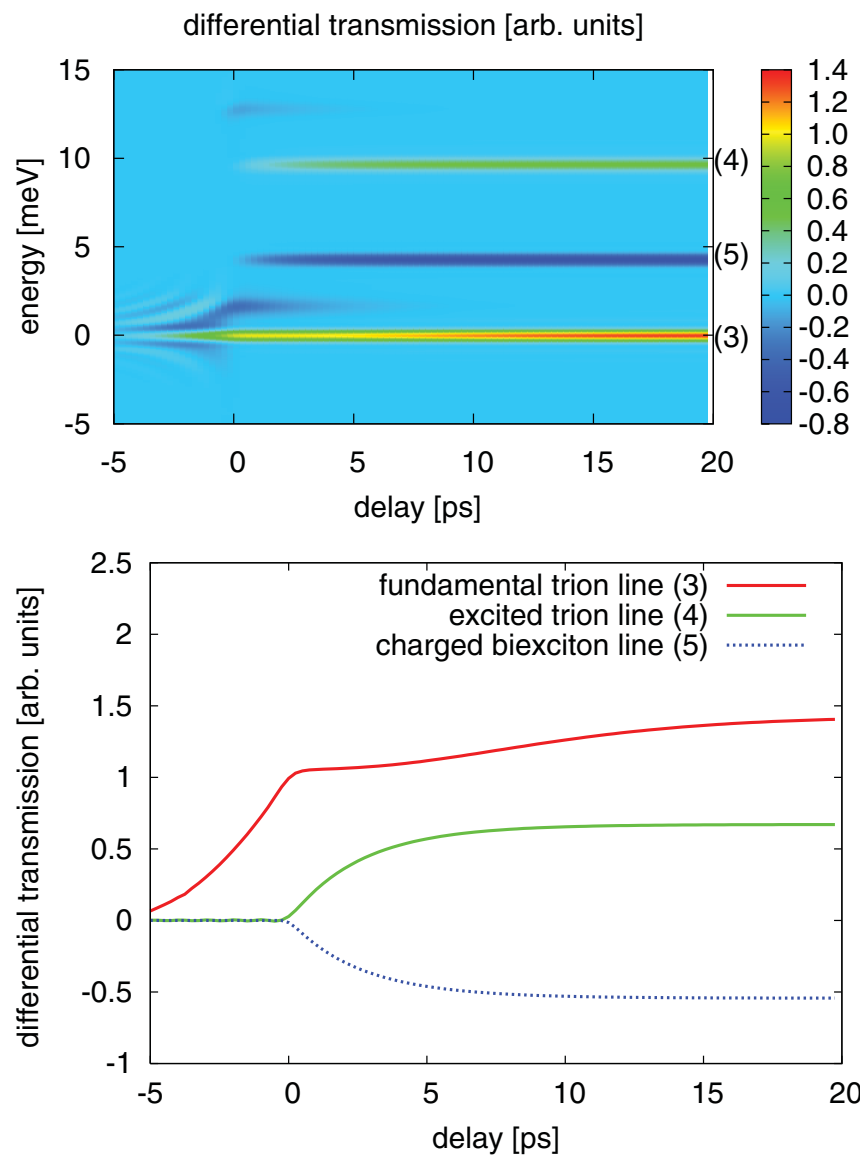

FIG. 8. (Color online) Upper panel: calculated differential transmission change $\Delta T / T$ as a function of the delay time $\tau$ between pump and probe pulse. The excitation conditions are the same as used in Fig. 6(b). Lower panel: cross section through the three main transition lines [(3), (4), and (5)]. 
after much longer times when also the electrons in the $S_{z}^{e}= \pm 1$ spin configurations have completely relaxed into the $s$ shell. Hence, we find that the shape of the probe pulse absorption in the spectral range of the lowest trion transition results from an interplay between Coulomb renormalizations and carrier relaxations and in large time windows strongly deviates from respective results obtained without taking into account Coulomb correlations.

In order to compare the pump-probe signal discussed in Fig. 6(b) with the experiment, ${ }^{24}$ we have calculated the corresponding differential transmission change $\Delta T / T=$ ( $\left.T_{\text {pump on }}-T_{\text {pump off }}\right) /\left|T_{\text {pump off }}\right|$ as measured in the experiment. The corresponding results are shown in Fig. 8. All five transmission lines as well as the spectral oscillations are visible and the normalized amplitude of the three main transition lines [(3), (4), and (5)] have pronounced amplitudes (see Fig. 8, lower panel). Therefore we predict a distinct red shifted gain and absorption line with an amplitude twice as large as on the FTL [see also Fig. 6(b)]. Both build-up times are given by the hole relaxation time of the trion system. These lines have not been seen in the experiment, ${ }^{24}$ because they were outside the investigated spectral window.

\section{CONCLUSIONS}

We have analyzed the influence of Coulomb interactions and carrier relaxation on the lowest energy transition of a single negatively charged QD. For this purpose, we have calculated pump-probe signals where the pump pulse excited resonantly a higher shell while the probe pulse was tuned to the lowest trion absorption. The considered pump and probe pulse resonances have been chosen according to states excited in the two-color pump-probe experiment of Ref. 24. We have discussed the role of various carrier spin configurations in the relaxation kinetics due to the interaction with the environment and we have shown, in agreement with the experiment, that the probed fundamental trion absorption line is noticeably modified as soon as the pump pulse generates an electron-hole pair in a higher shell. We traced back the origin of the experimentally reported instantaneous bleaching of the FTL absorption and the appearance of spectral oscillations for negative delay times to instantaneous Coulomb renormalizations that shift and split the lowest trion absorption line. We have compared the probe pulse-induced absorption in the presence and absence of Coulomb interactions and were able to show that Coulomb renormalization effects lead to much richer spectra. In addition to the differential transmission line observed in the experiment, our calculations predict new transitions with build-up and decay times related to the hole and electron relaxation times. Therefore an observation of the temporal evolution of these gain or absorption lines should provide detailed information on the carrier relaxation processes.

\section{ACKNOWLEDGMENTS}

This work was partly supported by a Research Group Linkage Project between the Münster and Wrocław groups funded by the Alexander von Humboldt Foundation. J. H. wishes to thank the DAAD for financial support.

\section{APPENDIX: EXTRACTING THE PUMP-PROBE SIGNAL}

The electric field describing both pump and probe pulse is given by

$$
\mathbf{E}(t)=\mathbf{E}_{\text {pump }}(t) e^{-i \omega_{1} t}+\mathbf{E}_{\text {probe }}(t-\tau) e^{-i \omega_{2}(t-\tau)} e^{i \phi},
$$

where $\omega_{1}$ and $\omega_{2}$ are the central frequencies of the pulses, $\mathbf{E}_{\text {pump }}(t)$ and $\mathbf{E}_{\text {probe }}(t)$ are the respective temporal envelopes, $\tau$ is the delay time, and $\phi$ is a relative phase between the pulses. This relative phase results, e.g., from different propagation directions $\mathbf{k}_{1}$ and $\mathbf{k}_{2}$ of the pulses; then it is given by $\left(\mathbf{k}_{1}-\right.$ $\left.\mathbf{k}_{2}\right) \cdot \mathbf{r}$. The excitation by this pulse pair generates the optical polarization

$$
\mathbf{P}(t, \tau, \phi)=\sum_{i j} \mathbf{M}_{i j} \rho_{i j}(t, \tau, \phi) .
$$

The pump-probe signal is then given by the optical polarization $\mathbf{P}$ induced by the probe pulse, i.e., the contribution to $\mathbf{P}$ with the same phase dependence $e^{i \phi}$. Following Ref. 33, it can be obtained by expanding the total optical polarization in a Fourier series according to

$$
\begin{gathered}
\mathbf{P}(t, \tau, \phi)=\sum_{n} \mathbf{P}_{n}(t, \tau) e^{i n \phi}, \\
\mathbf{P}_{n}(t, \tau)=\frac{1}{2 \pi} \int_{0}^{2 \pi} d \phi \mathbf{P}(t, \tau, \phi) e^{-i n \phi} .
\end{gathered}
$$

The desired pump-probe signal is then given by $\mathbf{P}_{\text {probe }}(t, \tau)=$ $\mathbf{P}_{n=1}(t, \tau)$. Technically, we perform a discrete Fourier transform of Eq. (A4) with $\phi_{n}=2 \pi n / m$ and $m=5$ that has been proven to be sufficient to calculate the pumpprobe signal. The probe pulse-induced absorption $\alpha \sim$ $\operatorname{Im}\left[\mathbf{P}_{\text {probe }}(\omega, \tau)\right] / \mathbf{E}_{\text {probe }}(\omega, \tau)$ is then obtained by a Fourier transform of $\mathbf{P}_{\text {probe }}(t, \tau)$ with respect to $t$.
${ }^{1}$ E. Biolatti, R. C. Iotti, P. Zanardi, and F. Rossi, Phys. Rev. Lett. 85, 5647 (2000).

${ }^{2}$ A. Imamoḡlu, D. D. Awschalom, G. Burkard, D. P. DiVincenzo, D. Loss, M. Sherwin, and A. Small, Phys. Rev. Lett. 83, 4204 (1999).

${ }^{3}$ M. Feng, I. D’Amico, P. Zanardi, and F. Rossi, Phys. Rev. A 67, 014306 (2003).
${ }^{4}$ X. Li, Y. Wu, D. Steel, D. Gammon, T. H. Stievater, D. S. Katzer, D. Park, C. Piermarocchi, and L. J. Sham, Science 301, 809 (2003).

${ }^{5}$ T. P. Spiller, I. D'Amico, and B. W. Lovett, New J. Phys. 9, 20 (2007).

${ }^{6}$ P. Michler, A. Kiraz, C. Becher, W. V. Schoenfeld, P. M. Petroff, L. Zhang, E. Hu, and A. Imamoglu, Science 290, 2282 (2000). 
${ }^{7}$ Z. Yuan, B. E. Kardynal, R. M. Stevenson, A. J. Shields, C. J. Lobo, K. Cooper, N. S. Beattie, D. A. Ritchie, and M. Pepper, Science 295, 102 (2002).

${ }^{8}$ P. Borri, W. Langbein, S. Schneider, U. Woggon, R. L. Sellin, D. Ouyang, and D. Bimberg, Phys. Rev. B 66, 081306 (2002).

${ }^{9}$ T. H. Stievater, X. Li, D. G. Steel, D. Gammon, D. S. Katzer, D. Park, C. Piermarocchi, and L. J. Sham, Phys. Rev. Lett. 87, 133603 (2001).

${ }^{10}$ T. Grange, E. A. Zibik, R. Ferreira, G. Bastard, B. A. Carpenter, P. J. Phillips, D. Stehr, S. Winnerl, M. Helm, M. J. Steer, M. Hopkinson, J. W. Cockburn, M. S. Skolnick, and L. R. Wilson, New J. Phys. 9, 259 (2007).

${ }^{11}$ E. A. Zibik, T. Grange, B. A. Carpenter, N. E. Porter, R. Ferreira, G. Bastard, D. Stehr, S. Winnerl, M. Helm, H. Y. Liu, M. S. Skolnick, and L. R. Wilson, Nat. Mater. 8, 803 (2009).

${ }^{12}$ M. V. Gurudev Dutt, J. Cheng, B. Li, X. Xu, X. Li, P. R. Berman, D. G. Steel, A. S. Bracker, D. Gammon, S. E. Economou, Ren-Bao Liu, and L. J. Sham, Phys. Rev. Lett. 94, 227403 (2005).

${ }^{13}$ A. Greilich, R. Oulton, E. A. Zhukov, I. A. Yugova, D. R. Yakovlev, M. Bayer, A. Shabaev, A. L. Efros, I. A. Merkulov, V. Stavarache, D. Reuter, and A. Wieck, Phys. Rev. Lett. 96, 227401 (2006).

${ }^{14}$ I. A. Yugova, M. M. Glazov, E. L. Ivchenko, and A. L. Efros, Phys. Rev. B 80, 104436 (2009).

${ }^{15}$ M. M. Glazov, I. A. Yugova, S. Spatzek, A. Schwan, S. Varwig, D. R. Yakovlev, D. Reuter, A. D. Wieck, and M. Bayer, Phys. Rev. B 82, 155325 (2010).

${ }^{16}$ M. Kugler, T. Andlauer, T. Korn, A. Wagner, S. Fehringer, R. Schulz, M. Kubová, C. Gerl, D. Schuh, W. Wegscheider, P. Vogl, and C. Schüller, Phys. Rev. B 80, 035325 (2009).

${ }^{17} \mathrm{X}$. Xu, B. Sun, P. R. Berman, D. G. Steel, A. S. Bracker, D. Gammon, and L. J. Sham, Science 317, 929 (2007).

${ }^{18}$ M. Kroner, A. O. Govorov, S. Remi, B. Biedermann, S. Seidl, A. Badolato, P. M. Petroff, W. Zhang, R. Barbour, B. D. Gerardot, R. J. Warburton, and K. Karrai, Nature (London) 451, 311 (2008).

${ }^{19}$ M. Wesseli, C. Ruppert, S. Trumm, H. J. Krenner, J. J. Finley, and M. Betz, Appl. Phys. Lett. 88, 203110 (2006).

${ }^{20}$ G. Wrigge, I. Gerhardt, J. Hwang, G. Zumofen, and V. Sandoghdar, Nat. Phys. 4, 60 (2007).

${ }^{21}$ M. Zecherle, C. Ruppert, E. C. Clark, G. Abstreiter, J. J. Finley, and M. Betz, Phys. Rev. B 82, 125314 (2010).

${ }^{22}$ T. Guenther, C. Lienau, T. Elsaesser, M. Glanemann, V. M. Axt, T. Kuhn, S. Eshlaghi, and A. D. Wieck, Phys. Rev. Lett. 89, 057401 (2002).

${ }^{23}$ T. Unold, K. Mueller, C. Lienau, T. Elsaesser, and A. D. Wieck, Phys. Rev. Lett. 92, 157401 (2004).

${ }^{24}$ F. Sotier, T. Thomay, T. Hanke, J. Korger, S. Mahapatra, A. Frey, K. Brunner, R. Bratschitsch, and A. Leitenstorfer, Nat. Phys. 5, 352 (2009).

${ }^{25}$ E. Biolatti, I. D’Amico, P. Zanardi, and F. Rossi, Phys. Rev. B 65, 075306 (2002).

${ }^{26}$ A. Wojs and P. Hawrylak, Solid State Commun. 100, 487 (1996).

${ }^{27}$ N. Baer, P. Gartner, and F. Jahnke, Eur. Phys. J. B 42, 231 (2004).

${ }^{28}$ A. Hartmann, Y. Ducommun, E. Kapon, U. Hohenester, and E. Molinari, Phys. Rev. Lett. 84, 5648 (2000).
${ }^{29}$ G. A. Narvaez and P. Hawrylak, Phys. Rev. B 61, 13753 (2000).

${ }^{30}$ M. Bayer, O. Stern, P. Hawrylak, S. Fafard, and A. Forchel, Nature (London) 405, 923 (2000).

${ }^{31}$ S. G. Schirmer and A. I. Solomon, Phys. Rev. A 70, 022107 (2004).

${ }^{32}$ G. Lindblad, Commun. Math. Phys. 48, 119 (1976).

${ }^{33}$ H. Haug and A. P. Jauho, Quantum Kinetics in Transport and optics of Semiconductors (Springer-Verlag, Berlin, 1996).

${ }^{34}$ O. Madelung, Semiconductors: Data Handbook (Springer-Verlag, Berlin, 2004).

${ }^{35}$ G. Bester and A. Zunger, Phys. Rev. B 68, 073309 (2003).

${ }^{36}$ S. Rodt, A. Schliwa, K. Pötschke, F. Guffarth, and D. Bimberg, Phys. Rev. B 71, 155325 (2005).

${ }^{37}$ M. Ediger, G. Bester, A. Badolato, P. M. Petroff, K. Karrai, A. Zunger, and R. J. Warburton, Nat. Phys. 3, 774 (2007).

${ }^{38}$ A. Schliwa, M. Winkelnkemper, and D. Bimberg, Phys. Rev. B 79, 075443 (2009).

${ }^{39}$ V. Mlinar, M. Bozkurt, J. M. Ulloa, M. Ediger, G. Bester, A. Badolato, P. M. Koenraad, R. J. Warburton, and A. Zunger, Phys. Rev. B 80, 165425 (2009).

${ }^{40}$ M. E. Ware, E. A. Stinaff, D. Gammon, M. F. Doty, A. S. Bracker, D. Gershoni, V. L. Korenev, Ş. C. Bădescu, Y. Lyanda-Geller, and T. L. Reinecke, Phys. Rev. Lett. 95, 177403 (2005).

${ }^{41}$ M. Z. Maialle, E. A. de Andrada e Silva, and L. J. Sham, Phys. Rev. B 47, 15776 (1993).

${ }^{42}$ S. V. Gupalov, E. L. Ivchenko, and A. V. Kavokin, J. Exp. Theor. Phys. 86, 388 (1998).

${ }^{43}$ M. M. Glazov and V. D. Kulakovskii, Phys. Rev. B 79, 195305 (2009).

${ }^{44}$ T. Stauber and R. Zimmermann, Phys. Rev. B 73, 115303 (2006).

${ }^{45}$ J. Puls, M. Rabe, H. J. Wünsche, and F. Henneberger, Phys. Rev. B 60, 16303 (1999).

${ }^{46}$ I. A. Akimov, T. Flissikowski, A. Hundt, and F. Henneberger, Phys. Status Solidi A 201, 412 (2004).

${ }^{47}$ H. Htoon, D. Kulik, O. Baklenov, A. L. Holmes Jr, T. Takagahara, and C. K. Shih, Phys. Rev. B 63, 241303 (2001).

${ }^{48}$ B. Alèn, K. Karrai, R. J. Warburton, F. Bickel, P. M. Petroff, and J. Martínez-Pastor, Physica E 21, 395 (2004).

${ }^{49}$ A. V. Khaetskii and Y. V. Nazarov, Phys. Rev. B 61, 12639 (2000).

${ }^{50}$ Ş. C. Bădescu, Y. B. Lyanda-Geller, and T. L. Reinecke, Phys. Rev. B 72, 161304 (2005).

${ }^{51}$ K. V. Kavokin, Phys. Rev. B 69, 075302 (2004).

${ }^{52}$ Ş. C. Bădescu and T. L. Reinecke, Phys. Rev. B 75, 041309 (2007).

${ }^{53}$ B. Fluegel, N. Peyghambarian, G. Olbright, M. Lindberg, S. W. Koch, M. Joffre, D. Hulin, A. Migus, and A. Antonetti, Phys. Rev. Lett. 59, 2588 (1987).

${ }^{54}$ M. Joffre, D. Hulin, A. Migus, A. Antonetti, C. Benoit à la Guillaume, N. Peyghambarian, M. Lindberg, and S. W. Koch, Opt. Lett. 13, 276 (1988).

${ }^{55}$ J. P. Sokoloff, M. Joffre, B. Fluegel, D. Hulin, M. Lindberg, S. W. Koch, A. Migus, A. Antonetti, and N. Peyghambarian, Phys. Rev. B 38, 7615 (1988).

${ }^{56}$ M. Lindberg and S. W. Koch, Phys. Rev. B 38, 7607 (1988).

${ }^{57}$ S. W. Koch, N. Peyghambarian, and M. Lindberg, J. Phys. C 21, 5229 (1988). 